\title{
A Boosting Based Approach for Automatic Micro-calcification Detection
}

\author{
Arnau Oliver ${ }^{1}$, Albert Torrent ${ }^{1}$, Meritxell Tortajada ${ }^{1}$, Xavier Lladó $^{1}$, \\ Marta Peracaula ${ }^{1}$, Lidia Tortajada ${ }^{2}$, Melcior Sentís ${ }^{2}$, and Jordi Freixenet ${ }^{1}$ \\ 1 Dept. Computer Architecture and Technology, University of Girona, Girona, Spain \\ aoliver@atc.udg.edu \\ 2 Radiology Dept, UDIAT-Centre Diagnòstic, Corporació Parc Taulí, Sabadell, Spain
}

\begin{abstract}
In this paper we present a boosting based approach for automatic detection of micro-calcifications in mammographic images. Our proposal is based on using local features extracted from a bank of filters for obtaining a description of the different micro-calcifications morphology. The approach performs an initial training step in order to automatically learn and select the most salient features, which are subsequently used in a boosting classifier to perform the detection. The validity of our method is demonstrated using 112 mammograms of the well-known digitised MIAS database and 280 mammograms of a full-field digital database. The experimental evaluation is performed in terms of ROC analysis, obtaining $A z=0.88$ and $A z=0.90$ respectively, and FROC analysis. The obtained results show the feasibility of our approach for detecting micro-calcifications in both digitised and digital technologies.
\end{abstract}

\section{Introduction}

Breast cancer continues to be a significant health problem in the world. It constitutes the most common cancer among women in the European Union [1], and it is estimated that in the United States between one in eight and one in twelve women will develop breast cancer during their lifetime 2 3. Mammography is the most effective and reliable method for an early detection of breast cancer which is fundamental for improving prognosis 4,5]. Mammographic images are characterised by high spatial resolution allowing the detection of subtle scale signs such as micro-calcifications and masses. In this work, we focused on the detection of micro-calcifications, which are tiny granular deposits of calcium that generally appear in a mammogram as small bright spots within an inhomogeneous background.

The automatic detection of micro-calcifications is a well-known topic in mammography, as can be seen in the different surveys covering this topic [6] or the recent works of Chang et al. [8, Nunes et al. [9] and Papadopoulos et al. [10]. The approaches for micro-calcification detection are usually based on two steps. Firstly the detection of suspicious regions is performed, usually tuning the algorithm parameters in order to detect as many suspicious regions as possible (i.e., detecting the largest number of micro-calcifications but also increasing the 
probabilities to detect normal tissue as being a micro-calcification). Secondly, a false positive reduction algorithm is applied in order to find those detected suspicious regions being in fact normal tissue. However, none of these microcalcification detection approaches have emerged as a standard algorithm.

It is well known that digital mammography allows to improve the detection of micro-calcifications thanks to its superior sensitivity [11. Unfortunately, this technology is not available in many countries and clinical centres due to its expensive cost. Therefore, reliable automatic approaches able to detect microcalcifications in film plates are still necessary. In this work we present a boosting based approach for the detection of micro-calcifications in both digital and digitised databases. Our proposal is based on learning the different morphology of the micro-calcifications using local features, which are extracted using a bank of filters. Afterwards, this set of features is used to train a pixel-based boosting classifier which at each round automatically selects the most salient one. Therefore, when a new mammogram is tested only the salient features are computed and used to classify each pixel of the mammogram as being part of a micro-calcification or actually being normal tissue.

The rest of this paper is structured as follows. The following section describes the proposed approach. Section 3 explains the methodology followed to perform the experimental evaluation, which is done using two different databases and ROC and FROC analysis. Finally, the paper ends with the conclusions and further work.

\section{Micro-calcification Detection}

The presented approach for micro-calcification detection is based on the work of Murphy et al. 12] for object detection using local features and boosting classifier. As is shown in Fig. 1, the proposed approach is divided in three parts. Firstly, we create a visual word dictionary, which is composed by convolving patches containing a micro-calcification with a bank of filters. Afterwards, the training data is found by convolving positive samples (patches containing a micro-calcification) and negative samples (patches of other tissues) with the words of the dictionary defined as the duple patch-filter. Finally, new mammograms are classified pixelby-pixel by the trained classifier. Hence, the detection problem is translated to a pixel-based classification approach.

In the following subsections we describe in more detail the three parts of our approach.

\subsection{Building the Dictionary}

The first task of the system consists in building the feature dictionary. This dictionary is similar to an atlas, since it contains samples (patches) of microcalcifications. However, it also contains the convolution of these patches with a bank of filters, including the delta function (which gives the own patch as a result), 4 Gaussian derivatives, a Laplacian filter, a corner detector, and 2 Sobel 


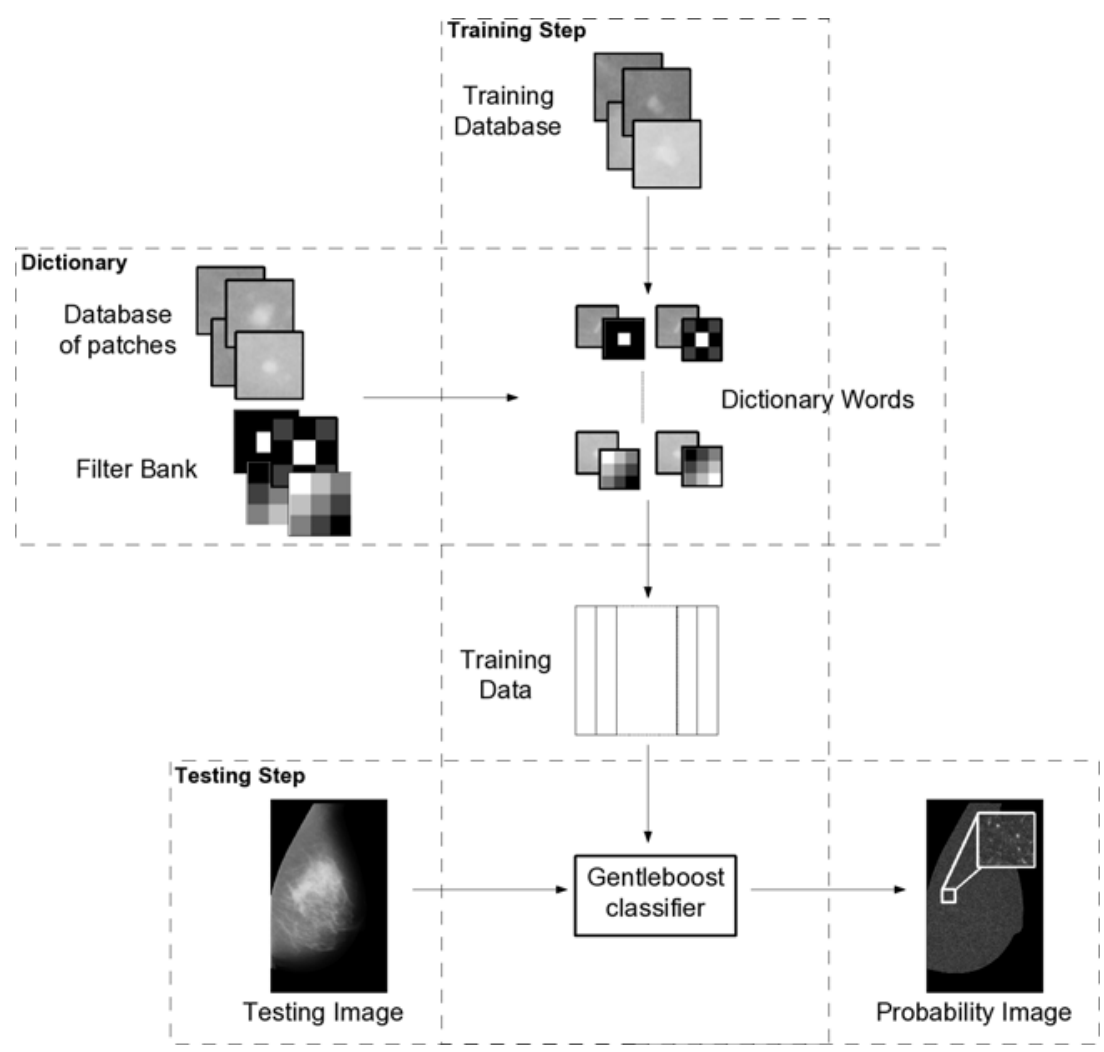

Fig. 1. Schematic representation of our approach. Note that the final result of the algorithm is a probability image where brighter pixels represent more confidence to be a micro-calcification.

filters. Hence, the dictionary contains grey-level and gradient information of the micro-calcifications and their neighbourhood.

Therefore, each dictionary word $w_{i j}$ can be understood as the duple $\left(p_{i}, f_{j}\right)$, where $p_{i}$ represents the patch and $f_{j}$ the filter.

\subsection{Training Step}

Once the dictionary is built, the words are used to extract the mammographic features that will be used for the micro-calcification detection. Hence, for the training step, we need a different database of patches containing instances of both patches with micro-calcifications and patches from the rest of the mammogram tissues.

The feature extraction of each training image patch consists in two operations. Firstly, it is convolved with all the bank filters, and secondly, the normalised 
cross-correlation with all the words is computed. Mathematically, both operations can be summarised as:

$$
v=\left(I * f_{j}\right) \otimes w_{i j}
$$

where $I$ is the training image patch convolved $(*)$ with the filter $f_{j}$ and crosscorrelated $(\otimes)$ with the word $w_{i j}$ (note that $w_{i j}=p_{i} * f_{j}$ ). The resulting value $v$ represents the similarity of the training patch and the dictionary word. Therefore, for each training image patch, a vector of features $v$ is constructed by crosscorrelating all the dictionary words $w_{i j}$ with the convolution of the patch itself with filter $f_{j}$. Notice here the necessity of keeping the filter as well as the patch in the dictionary word.

In contrast with the original approach that uses all pixels in the image as the centre of a positive or a negative patch, we manually select these points in each training image. In particular, we select the centre of the micro-calcifications as positive training examples and some random locations of the background containing examples of different tissues as negative training examples. Note that this is necessary in order to reduce the high computational cost due to the large size of the mammograms.

At this point, the positive and negative training examples have been characterised. Therefore, this data can be used to train a classifier. In this work, we have used the Gentleboost algorithm 13 .

Boosting algorithms are based on the simple idea that the sum of weak classifiers can produce a strong classifier. In the Gentleboost algorithm, the weak classifiers $\left(h_{t}\right)$ are simple regression stumps with one of the features, so at each round $t$ the feature with less error is selected. The weak classifier used is:

$$
h_{t}(x)=a \delta\left(x_{i}>t h\right)+b
$$

where th is a threshold that determines if pattern $x$ belongs to the object class, $x_{i}$ is the $i$ 'th dimension of $x$, and $a$ and $b$ are parameters selected to minimise the error of the classifier ( $a$ is the regression slope and $b$ the offset):

$$
e=\sum\left(z\left(y-\left(a\left(x_{i}>t h\right)+b\right)\right)^{2}\right)
$$

At each round the training data weights $(z)$ are updated, increasing in the following round the possibility of classifying correctly the previous incorrectly classified points. In the GentleBoost algorithm the data weights are updated:

$$
z_{t+1}=z_{t} e^{y \cdot h_{t}(x)}
$$

Hence, when testing a new data, the final (strong) classifier is computed using the weak classifier created at each round of the boosting. Therefore, the testing data is classified according to the sign of the sum of weak classifiers:

$$
H(x)=\sum h(x)
$$

The absolute value of $H(x)$ shows the confidence of the classified data. 


\subsection{Testing Step}

Once the classifier is built, the system is ready for the testing step, where the strong classifier $H(x)$ is applied to new images in order to evaluate the microcalcifications detection. Note that the classifier is pixel-based, i.e. it is applied one-by-one to all the image pixels. Therefore, the result of our approach after evaluating a mammogram is a probability image, where high values represents more confidence to be a micro-calcification.

Since the classifier is pixel-based, a pre-processing step may be necessary in order to avoid the algorithm detecting micro-calcifications in the background (in digitised images) and in the pectoral muscle. In particular, we used a previous developed algorithm to detect the skin-line border 14 and the approach of Kwok et al. 15] to remove the pectoral muscle.

\section{Results}

The experimental results were performed using two different subsets of mammograms. The first subset of 112 mammograms was extracted from the MIAS database [16, and contained all the mammograms with micro-calcifications (22 in total) and a set of 90 normal mammograms. In order to train the classifier with positive examples, an expert accurately marked among 5 and 15 micro-calcifications in each mammogram containing micro-calcifications, while the negative examples were randomly obtained from the rest of tissues of normal mammograms. On the other hand, we also used a set of 280 full-field digital mammograms extracted from a non-public database, 90 of them containing microcalcifications and 190 being normal ones. The mammograms were acquired using a Hologic Selenia mammograph, with resolution 70 micron-pixel, size 4096×3328, and 12-bit depth. The selection of the training points was performed in the same way that using the MIAS subset.

In order to perform the evaluation of our experiments in both databases we used a 10-folder cross-validation methodology. Therefore, we divided both datasets in 10 different groups. One of the groups was used to create the dictionary, eight of them were merged for training the system, while the remaining one was used for testing it. This procedure was repeated until all groups were used for testing. Hence, each mammogram appears in the test set only once.

To perform the quantitative evaluation we used Receiver Operating Characteristic (ROC) and Free-response Receiver Operating Characteristic (FROC) analysis. In ROC analysis, a graphical curve represents the true positive rate (number of detected mammograms with micro-calcifications divided by the total number of mammograms with micro-calcifications) as a function of the false positives rate (number of normal mammograms incorrectly detected as containing micro-calcifications divided by the total number of normal mammograms). Moreover, the percentage value under the curve $\left(A_{z}\right)$ is an indication for the overall performance of the observer, and is typically used to analyse the performance of the algorithms. Note that points in the curve are obtained by thresholding at different levels the result of Eq. 5 . 


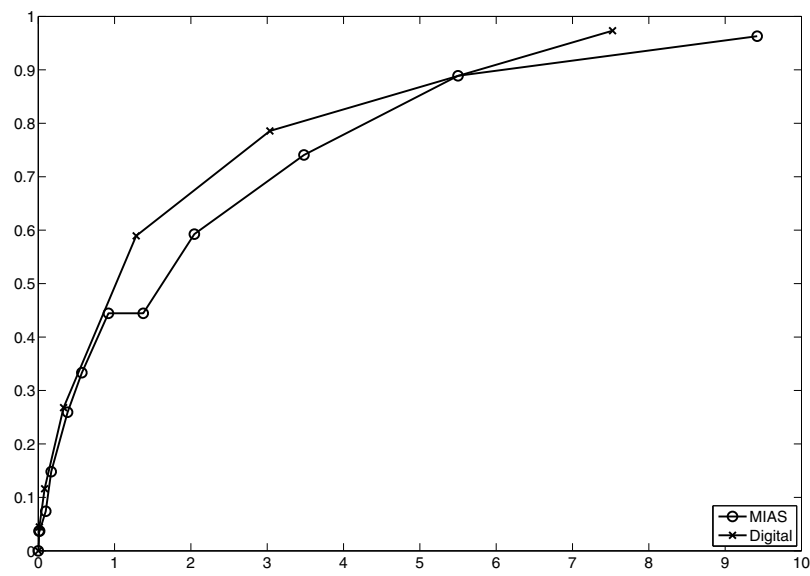

Fig. 2. Obtained FROC when testing the MIAS and the digital database. Note that we obtained better results when testing the latter.

On the other hand, in FROC analysis the Lesion Localisation Fraction (LLF) is obtained as the number of correctly detected lesions relative to the total number of lesions and the Non-Lesion Localisation Fraction as the number of non-correctly detected lesions relative to the total number of images. The FROC curve is the graphical summary of both measures [17. Note that the definition of a detected region is needed. In this paper we assume that a region is detected if a set of suspicious points are detected inside the region marked by the experts.

Using ROC analysis, we achieved an area under the ROC of $A z=0.88$ for the MIAS subset and $A z=0.90$ for the digital database. Note here the benefits of the digital technology, since applying the same method it allows a better detection even when using a bigger number of images for testing.

On the other hand, the obtained FROC curves when testing both databases are shown in Fig. 2. For the MIAS database we obtained 4.3 false positives per image at a sensitivity of $80 \%$, while using the digital database we obtained 3.2 false positives per image at the same sensitivity. Note that these numbers can be decreased using a posterior false positive step. For instance, introducing spatial constraints, and hence assuming that the micro-calcifications appear in the form of clusters. Comparing the performance of the approach using both databases we noticed that again we obtained better results using the digital one. This is the expected behaviour since digital mammography improves the contrast between the different internal structures.

Finally, we compare in Table 1 the results presented in this paper with those obtained by different current state-of-the-art approaches using ROC analysis. Note that each approach used a different subset of images coming also from different databases and hence the comparison is only done in a qualitative way. Note that the obtained results using our approach are of the same order as the 
Table 1. Comparison of the obtained experimental results with state-of-the-art algorithms for micro-calcification detection i mammographic images

\begin{tabular}{|c||c|c|}
\hline Authors & Cases & Results $\left(A_{z}\right)$ \\
\hline \hline Chang et al. (2008) $[8]$ & 194 & 0.90 \\
\hline Nunes et al. (2007) $[9]$ & 121 & 0.93 \\
\hline Papadopoulos et al. (2008) 10$]$ & 60 & 0.92 \\
\hline \hline Our approach - MIAS & $\mathbf{1 1 2}$ & $\mathbf{0 . 8 8}$ \\
\hline Our approach - Digital & $\mathbf{2 8 0}$ & $\mathbf{0 . 9 0}$ \\
\hline
\end{tabular}

ones shown in the table. However, we want to stress here that our approach, in contrast with the other ones, does not include a false positive reduction step, and hence they are obtained directly from the detection step. Hence, applying such false positive reduction step would probably increase them.

\section{Conclusion}

We have presented a new approach for micro-calcification detection based on extracting local features for characterising the morphology of the microcalcifications. The proposed boosting approach allows the selection of the most salient features at each round, reducing the computational time of the testing step. The performed experiments have shown the validity of our proposal when using either digitised or digital mammograms.

Further work is focused in two directions. Firstly, we would like to integrate a false positive reduction step into the boosting algorithm to improve the results. Secondly, we also want to expand this work for detecting and diagnosing clusters of micro-calcifications.

\section{Acknowledgement}

This work was supported in part by Ministerio de Educación y Ciencia of Spain under Grant TIN2007-60553 and by the CIRIT and CUR of DIUiE of Generalitat de Catalunya under Grant 2008SALUT00029.

\section{References}

1. Eurostat: Health statistics Atlas on mortality in the European Union. Office for Official Publications of the European Union (2002)

2. Bray, F., McCarron, P., Parkin, D.M.: The changing global patterns of female breast cancer incidence and mortality. Breast Cancer Research 6(6), 229-239 (2004)

3. American Cancer Society: Breast cancer: facts and figures. 2007-08. ACS (2007)

4. Sivaramakrishna, R., Gordon, R.: Detection of breast cancer at a smaller size can reduce the likelihood of metastatic spread: A quantitative analysis. Acad. Radiol. 4(1), 8-12 (1997) 
5. Sener, S.F., Winchester, D.J., Winchester, D.P., Barrera, E., Bilimoria, M., Brinkmann, E., Alwawi, E., Rabbitt, S., Schermerhorna, M., Du, H.: Survival rates for breast cancers detected in a community service screening mammogram program. Am. J. Surg. 191(3), 406-409 (2006)

6. Cheng, H.D., Cai, X., Chen, X., Hu, L., Lou, X.: Computer-aided detection and classification of microcalcifications in mammograms: a survey. Pattern Recogn. 36(12), 2967-2991 (2003)

7. Rangayyan, R.M., Ayres, F.J., Desautels, J.E.L.: A review of computer-aided diagnosis of breast cancer: Toward the detection of subtle signs. J. Frankl. Inst. 344(3-4), 312-348 (2007)

8. Chang, T.T., Feng, J., Liu, H.W., Ip, H.H.S.: Clustered microcalcification detection based on a multiple kernel support vector machine with grouped features. In: IAPR Int. Conf. Pattern Recogn., pp. 1-4 (2008)

9. Nunes, F.L.S., Schiabel, H., Goes, C.E.: Contrast enhancement in dense breast images to aid clustered microcalcifications detection. J. Digit. Imaging 1(20), 53-66 (2007)

10. Papadopoulos, A., Fotiadis, D.I., Costaridou, L.: Improvement of microcalcification cluster detection in mammography utilizing image enhancement techniques. Comput. Biol. Med. 10(38), 1045-1055 (2008)

11. Fischer, U., Baum, F., Obenauer, S., Luftner-Nagel, S., von Heyden, D., Vosshenrich, R., Grabbe, E.: Comparative study in patients with microcalcifications: fullfield digital mammography vs screen-film mammography. Epidemiol. Rev. 11(12), 2679-2683 (2002)

12. Murphy, K., Torralba, A., Eaton, D., Freeman, W.T.: Object detection and localization using local and global features. In: Sicily W. on Object Recognition (2005)

13. Friedman, J., Hastie, T., Tibshirani, R.: Additive logistic regression: a statistical view of boosting. Ann. Stat. 38(2), 337-374 (2000)

14. Martí, R., Oliver, A., Raba, D., Freixenet, J.: Breast skin-line segmentation using contour growing. In: Martí, J., Benedí, J.M., Mendonça, A.M., Serrat, J. (eds.) IbPRIA 2007. LNCS, vol. 4478, pp. 564-571. Springer, Heidelberg (2007)

15. Kwok, S.M., Chandrasekhar, R., Attikiouzel, Y., Rickard, M.T.: Automatic pectoral muscle segmentation on mediolateral oblique view mammograms. IEEE Trans. Med. Imag. 23(9), 1129-1140 (2004)

16. Suckling, J., Parker, J., Dance, D.R., Astley, S.M., Hutt, I., Boggis, C.R.M., Ricketts, I., Stamatakis, E., Cerneaz, N., Kok, S.L., Taylor, P., Betal, D., Savage, J.: The Mammographic Image Analysis Society digital mammogram database. In: Int. Work. Dig. Mammography, pp. 211-221 (1994)

17. Yoon, H.J., Zheng, B., Sahiner, B., Chakraborty, D.P.: Evaluating computer-aided detection algorithms. Med. Phys. 34(6), 2024-2038 (2007) 